\title{
Les différentes alternatives d'anticoagulation au couple héparine/protamine en chirurgie cardiaque sous circulation extra-corporelle
}

\section{Alternatives to heparin and protamine anticoagulation for cardiopulmonary bypass in cardiac surgery}

\author{
Aurore Bouraghda, MD • Pierre Gillois, MD, PhD • \\ Pierre Albaladejo, MD, PhD \\ Received: 30 September 2014 / Accepted: 6 February 2015/Published online: 20 February 2015 \\ (C) Canadian Anesthesiologists' Society 2015
}

\begin{abstract}
Résumé
Objectif L'héparine et la protamine constituent la stratégie d'anticoagulation de référence en chirurgie cardiaque sous circulation extra-corporelle (CEC), mais elle présente des limites d'utilisation. L'objectif principal de cette étude est de trouver des alternatives d'anticoagulation fiables en chirurgie cardiaque sous CEC. Les objectifs secondaires sont pour chaque molécule: de définir les avantages et inconvénients, de proposer un protocole d'utilisation et une analyse médico-économique.

Source Une revue systématique de la littérature fut réalisée entre septembre 2012 et décembre 2013. Elle s'est basée sur le protocole établi par la «Cochrane Collaboration Handbook». Vingt publications furent analysées. La base de données Thériaque du CHU de Grenoble a permis l'analyse économique.
\end{abstract}

Contribution des auteurs Aurore Bouraghda: auteur principal. Pierre Gillois: soutien méthodologique, soutien rédactionnel. Pierre Albaladejo: délivrance du sujet d'étude, soutien méthodologique et scientifique.

A. Bouraghda, MD

Pôle Anesthésie-Réanimation, UJF-Grenoble, Grenoble, France

\author{
A. Bouraghda, MD $(\bowtie)$ \\ Interne en Anesthésie - Réanimation, CHU de Grenoble, \\ Grenoble, France \\ e-mail: ABouraghda@chu-grenoble.fr \\ P. Gillois, $\mathrm{MD}, \mathrm{PhD}$ \\ Laboratoire TIMC UMR, Themas, Pôle de Santé Publique, \\ UJF-Grenoble, Grenoble, France \\ P. Albaladejo, MD, PhD \\ Laboratoire TIMC UMR 5525, Themas, Pôle d' \\ Anesthésie-Réanimation, UJF-Grenoble, Grenoble, France
}

\begin{abstract}
Constatations principales Sept alternatives d'anticoagulation furent retenues: le danaparö̈de sodique, la lépirudine, l'argatroban, la bivalirudine, l'ancrod, l'idraparinux et l'EP217609. Le danaparoüde sodique présente des variabilités individuelles. Les inhibiteurs directs de la thrombine sont prometteurs. Plusieurs études (EVOLUTION-ON, CHOOSE-ON) ont proposées un protocole d'utilisation fiable avec la Bivalirudine. L'Ancrod induisait une majoration des produits sanguins consommés. L'EP217609 est un anticoagulant de synthèse en étude de phase IIa. C'est un inhibiteur indirect du facteur Xa et un inhibiteur direct de la thrombine libre et liée, antagonisable par l'avidine.

Conclusion L'anticoagulant idéal pouvant être utilisé en chirurgie cardiaque sous CEC n'existe pas. L'héparine et de la protamine reste la stratégie d'anticoagulation de référence. La Bivalirudine est la molécule la plus prometteuse à ce jour malgré l'absence de stratégie d'antagonisation facile et un coût d'utilisation élevé.
\end{abstract}

\footnotetext{
Abstract

Purpose Heparin anticoagulation followed by protamine reversal is commonly used in cardiopulmonary bypass $(C P B)$ cardiac procedures, but this strategy has some limitations. The primary objective of this study was to determine the reliable alternatives for anticoagulation during CPB for cardiac surgery. For each drug proposed, the secondary objectives were to outline the main advantages and disadvantages, to propose a therapeutic protocol, and to provide a cost-benefit analysis.

Source A systematic review of the literature was performed between September 2012 and December 2013. It was based on the protocol established by the "Cochrane collaboration Handbook". Twenty articles were analyzed.
} 
The Theriaque database from the University Hospital of Grenoble made the economic analysis possible.

Principal findings Seven alternative anticoagulation strategies were considered: danaparoid sodium, lepirudin, argatroban, bivalirudin, ancrod, idraparinux, and EP217609. Danaparoid sodium has issues with individual variability. Several studies (EVOLUTION-ON, CHOOSE-ON) proposed a reliable therapeutic protocol for bivalirudin. Ancrod resulted in an increase in the transfusion of blood products. Direct thrombin inhibitors offer a promising alternative. EP217609 is a synthetic anticoagulant currently undergoing Phase II a clinical trials. It is an indirect inhibitor of factor Xa, a direct inhibitor of free and bound thrombin, and can be neutralized by avidin.

Conclusions The ideal anticoagulation strategy for cardiac surgery with $C P B$ does not exist. Heparin and protamine remain the gold standard for anticoagulation therapy. To date, bivalirudin is the most promising molecule despite its high cost and lack of a readily available antagonist.

Lors d'une intervention de chirurgie cardiaque, une anticoagulation optimale est indispensable pour prévenir la formation de caillots dans le circuit de circulation extra-corporelle (CEC) ainsi que les complications thromboemboliques. Depuis plus de 50 ans, l'héparine non fractionnée et son antidote, la protamine, reste la stratégie d'anticoagulation de référence en raison de nombreux atouts: ${ }^{1}$ coût peu élevé, délai d'action rapide, durée de vie courte, facilité de titration et de monitorage, réversion rapide par la protamine, absence de nécessité d'adaptation à la fonction rénale. Cependant, le couple héparine non fractionnée /protamine présente des limites d'utilisation. ${ }^{2}$ L'héparine non fractionnée pose le problème de la variabilité de réponse individuelle, de résistance à l'héparine, du développement d'anticorps anti-facteur 4 plaquettaire $(\mathrm{pF} 4)$ pouvant conduire à des thrombopénies induites par l'héparine. ${ }^{2}$ Son antidote, le sulfate de protamine n'est pas dénué d'effets indésirables ${ }^{3}$ notamment hémodynamiques (hypotension, bradycardie, vasodilatation périphérique, vasoconstriction pulmonaire, œdème pulmonaire non cardiogénique, ...) ou anaphylactiques. La présence de perturbations hémodynamiques après l'administration de protamine comme réversion de l'héparine lors de chirurgie cardiaque sous CEC ont été associées à un taux de mortalité accrue dans une étude de cohorte américaine. ${ }^{4}$

De plus, la révélation de scandale sanitaire, tel que l'héparine chinoise contaminée par le sulfate de chondroïtine sursulfaté ayant conduit à des réactions allergiques mortelles ${ }^{5}$ ou celui de la menace d'une possible pénurie en protamine après la catastrophe de Fukushima, ${ }^{6}$ pose la question d'alternatives anticoagulantes synthétiques et non plus naturelles fiables.

L'objectif principal de cette étude est de trouver une alternative fiable à la stratégie d'anticoagulation actuelle, qu'est le couple héparine/protamine en chirurgie cardiaque sous CEC. Les objectifs secondaires sont de mettre en évidence les bénéfices et les effets indésirables de chaque alternative thérapeutique connue, d'avoir une réflexion sur la sécurité de son utilisation et le cas échéant, de définir un protocole d'utilisation avec des posologies, un monitorage permettant une utilisation optimale de chaque molécule et enfin de définir le coût de l'utilisation pour chacune d'entre elles.

\section{Méthodes}

Un travail de recherche basé sur une revue systématique de la littérature a été réalisé entre septembre 2012 et décembre 2013. L'élaboration de cette revue systématique de la littérature s'est basée sur le protocole préalablement établi selon le « Cochrane Collaboration Handbook ».

Une revue systématique de la littérature nécessite une démarche scientifique rigoureuse constituée de plusieurs étapes:

1) chercher toutes les publications existantes, essais cliniques ou autres études appropriées, publiées ou non, qui ont répondu à la question posée.

2) évaluer la qualité de chaque étude et sélectionner celles qui correspondent à un standard de qualité élevé.

3) effectuer la synthèse des résultats obtenus d'après les études sélectionnées.

4) effectuer une analyse statistique en regroupant les résultats quantifiés provenant de plusieurs études en un résultat résumé.

L'objectif de ce travail a été défini préalablement: «rechercher une alternative fiable à la stratégie d'anticoagulation basée sur l'héparine et la protamine en chirurgie cardiaque sous circulation extra-corporelle ». Celui-ci a consisté à effectuer une recherche bibliographique médicale via des plateformes spécialisées: Pubmed, DOC'CISMEF, GAEL, EM-consulte, SFAR, Wikipedia, Science Direct.

Les mots-clefs MeSH suivants ont été utilisés: « anticoagulation », « ancrod », « argatroban », «bivalirudin», « cardiac surgical procedures», « cardiopulmonary bypass », « danaproïd », « heparin », « idraparinux », « iloprost », « lepirudin », « coronary artery bypass, off-pump », « protamines », « tirofiban ». 
Afin d'avoir une recherche exhaustive, les mots-clefs suivants ont été associés aux mots-clefs MeSH précédemment cités avec l'opérateur booléen « AND »: « alternative anticoagulation », «EP $217609 »$, « heparin-induced thrombocytopenia », « heparin removal device », « on-pump ». Les références bibliographiques ou citations ont été regroupées dans le logiciel de gestion de référence Endnote.

Cette revue systématique de la littérature a été complétée par une approche économique des différentes alternatives existantes étudiées. Cette étude économique a été réalisée à partir de la base de données Thériaque, recensant l'ensemble des médicaments disponible du $\mathrm{CHU}$ de Grenoble. L'estimation du coût de chaque stratégie a été basée pour une durée moyenne de CEC de $75 \mathrm{~min}^{8} \mathrm{chez}$ un patient de $60 \mathrm{~kg}$, d'après les protocoles d'utilisation utilisés dans les principales études.

\section{Résultats}

La recherche bibliographique a permis l'analyse de 35 publications concernant différentes alternatives existantes.

Les études se rapportant à des alternatives d'utilisation ne correspondant pas à l'objectif principal telles que la chirurgie à cœur battant, l'utilisation de circuits de CEC pré-héparinés ont été exclues de l'analyse.

Vingt publications ont été sélectionnées (essai multicentrique, prospectif, contrôlé, randomisé, étude de cohorte, série de cas et rapport de cas), originaire de différents pays (Tableau 1). Ces vingt publications concernent les alternatives d'anticoagulation les plus fréquemment citées dans les publications concernant ce sujet.

Définir une stratégie d'anticoagulation en chirurgie cardiaque nécessite de prendre en considérations plusieurs facteurs: ${ }^{1}$

- Premièrement, l'autorisation d'utilisation et la disponibilité de la molécule pour le praticien.

- Deuxièmement, la possibilité de monitorer de façon fiable et rapide l'action anticoagulante de la molécule. En effet, pour chaque molécule, un moyen de monitorage spécifique doit être privilégié [l'activité anti-Xa pour le danaparoide sodique, le temps d'écarine (ECT, ou test de coagulation à l'écarine), l'ECT pour les inhibiteurs directs de la thrombine (IDT),...].

- Troisièmement, les spécificités de l'état de santé du patient, comme la présence d'une insuffisance hépatique ou rénale, doivent être pris en compte dans le cadre d'une stratégie d'anticoagulation individualisée.
- Enfin, l'expérience de l'équipe soignante dans le maniement de la molécule n'est pas à négliger, de même que le coût économique de l'utilisation de nouvelles molécules.

Plusieurs molécules ont été utilisées comme alternative à la stratégie de référence, chacune d'entre elles présentent des avantages et des inconvénients qui vont être décrits.

Danaparoïde sodique

Le danaparoïde sodique est une préparation composée de glycosaminoglycanes sulfatés non héparinique de faible poids moléculaire provenant de la muqueuse intestinale du porc. Cette molécule possède une activité anticoagulante principalement par une inhibition du facteur Xa avec un ratio d'activité antiXa/antiIIa de 22:1. ${ }^{1}$ Sur le plan pharmacologique, la demi vie d'élimination de l'activité anti-Xa est longue (environ $25 \mathrm{~h}$ ). L'élimination se fait essentiellement par voie rénale. Aucun antagoniste fiable n'existe à l'heure actuelle, ce qui pose le problème des saignements massifs avec cette molécule. Depuis 1990, plusieurs auteurs ont étudié l'utilisation du danaparoïde sodique en chirurgie cardiaque sous CEC, notamment chez des patients ayant des antécédents de thrombopénie induite par l'héparine (TIH). ${ }^{9-11}$

Magnani et coll. ${ }^{12}$ ont proposé un schéma d'administration basé sur une injection de 8750 UI en bolus intraveineux après la sternotomie et avant la canulation, 7500 UI dans le volume d'amorçage de la CEC avec des injections horaires de $1500 \mathrm{U}$. La dernière injection devait être faite plus d'une heure avant la fin présumée de la CEC. La dose initiale en bolus pouvait être diminuée 5000 UI chez les patients de moins de $60 \mathrm{~kg}$ et augmentée à 10000 UI chez les patients de plus de $90 \mathrm{~kg}$.

En se basant, sur ce protocole, l'utilisation du danaparoïde sodique reviendrait à 271.5 euros par intervention (336.66 USD - 382.81 CAD). Ceci correspond à un coût moyen pour 75 min de CEC chez un patient de $60 \mathrm{~kg}$ contre 19,45 euros (15.7 USD - 27.4 CAD) pour la stratégie d'anticoagulation de référence (Tableau 2).

Ces études ont mis en avant plusieurs limites d'utilisation de cet anticoagulant: les différents case report publiés ne permettent pas de définir la dose optimale à administrer en raison de grandes variabilités individuelles avec à la fois des cas de saignements postopératoires graves, de transfusion massive, mais aussi d'arrêt prématuré de la chirurgie en raison de la formation de caillots dans le circuit de CEC. Les auteurs se sont également heurtés à la complexité du monitorage: l'évaluation du taux plasmatique de l'activité anti-Xa est la seule méthode de monitorage fiable du danaparoïde 
sodique, mais des variabilités existent en fonction du délai entre l'injection et le prélèvement sanguin, du poids du patient, de la fonction rénale et probablement d'autres facteurs. Ainsi une même dose pourra se traduire par différentes activités anti-Xa.

Il semblerait donc que bien que le danaparoïde sodique ait l'autorisation de mise sur le marché (AMM) chez les patients ayant des antécédents de TIH, ce ne soit pas la meilleure alternative à l'héparine dans la cadre de la chirurgie cardiaque, d'autant plus que la réactivité croisée avec les anticorps responsables de TIH n'est pas nulle (environ $17 \%)^{1}$

Les inhibiteurs directs de la thrombine

Il s'agit d'une famille d'anti-thrombotiques, qui inhibe la cascade de la coagulation au niveau de la thrombine. Ces molécules ont la capacité de se lier à la fois à la thrombine libre et à la thrombine liée. ${ }^{13}$ Trois molécules (la lépirudine, l'argatroban et la bivalirudine) sont autorisées par la FDA (Food and Drug Association) pour le traitement de la TIH compliquée de thrombose. ${ }^{13}$ Contrairement à l'héparine, les IDT n'interagissent pas avec les protéines plasmatiques, ce qui rend leur relation dose-effet plus prédictible $^{14}$ et explique qu'ils constituent une alternative prometteuse à l'utilisation d'héparine. Leur principal inconvénient reste l'absence d'antidote fiable à ce jour. La Figure schématise l'action des IDT au niveau de la cascade de coagulation. ${ }^{13}$

\section{Lépirudine}

La lépirudine est le premier inhibiteur direct de la thrombine, qui fut utilisé en CEC. Il s'agit d'une hirudine recombinante, dérivée de cellules de levures, qui se lie de façon hautement spécifique et irréversible au niveau de deux sites de la thrombine libre et lieee. ${ }^{15}$ Son action anti-thrombotique est indépendante de l'antithrombine III et elle n'est pas inhibée par le PF4.

La lépirudine est excrétée et métabolisée en quasi-totalité par le rein. ${ }^{15}$ Une adaptation de posologie à la clairance de la créatinine est donc justifiée et son utilisation doit être évitée en présence d'une insuffisance rénale. La demi-vie d'élimination plasmatique est d'environ $80 \mathrm{~min}$ chez le sujet sain ayant une fonction rénale normale mais peut dépasser les $120 \mathrm{~h}$ en cas d'altération sévère de la fonction rénale.

Elle possède une fenêtre thérapeutique étroite, avec des effets indésirables pouvant être graves: douleur au point de ponction, réaction allergique cutanée, saignement, réaction anaphylactique. ${ }^{15}$ Cette liste est non exhaustive. Le risque anaphylactique peut être gravissime avec plusieurs cas de choc anaphylactique fatal, particulièrement en cas de réexposition. ${ }^{16}$ Une surveillance rapprochée est de ce fait nécessaire.

\section{Argatroban}

L'argatroban constitue une alternative à l'héparine intéressante chez l'insuffisant rénal. En effet, cet IDT présente un métabolisme essentiellement hépatique avant d'être éliminé par le système gastro-intestinal. ${ }^{1} \mathrm{Sa}$ demi-vie plasmatique est relativement courte (39-51 min) et ne semble pas majorée en cas d'insuffisance rénale. ${ }^{1}$ Son action anticoagulante se fait via une liaison univalente réversible au niveau du site actif de la thrombine et ceci sans la nécessité de cofacteur contrairement à l'héparine. ${ }^{1}$ Le monitorage de son action se fait conventionnellement par la mesure du temps de coagulation activée (ACT). ${ }^{17}$ Actuellement l'argatroban a l'AMM pour le traitement des thrombopénies à l'héparine (TIH) aigues ainsi que les interventions coronariennes percutanées. Son utilisation lors d'intervention de chirurgie cardiaque sous CEC reste limitée à quelques séries de cas. $^{17-19}$ Sur le plan économique, son coût d'utilisation reste proche de celui de l'héparine: entre 23 et 46 euros par procédure (28-57 USD) (32-65 CAD) (Tableau 2).

\section{Bivalirudine}

La bivalirudine constitue l'une des alternatives à l'héparine la plus prometteuse à l'heure actuelle et fait l'objet de nombreuses publications. ${ }^{8,13,20-22}$ Il s'agit d'un petit peptide de 20 acides aminés, qui agit comme un inhibiteur non compétitif, direct et réversible de la thrombine. Cette molécule, dont l'administration est exclusivement intraveineuse, a un profil pharmacologique unique: elle a l'avantage d'avoir un effet anticoagulant quasi immédiat après injection avec une relation dose-effet linéaire en présence d'une fonction rénale normale, d'avoir une demi-vie courte (environ $30 \mathrm{~min}$ ) et d'être éliminée principalement par des protéases circulantes, avec uniquement $20 \%$ d'élimination rénale. ${ }^{8}$ Aucun antagoniste spécifique à ce traitement n'existe à l'heure actuelle. Chez les patients insuffisants rénaux, l'utilisation de l'hémodialyse s'avère efficace pour l'élimination de la molécule.

La bivalirudine se lie à deux régions distinctes de la thrombine: l'une est localisée au niveau du site catalytique et l'autre au niveau du site de liaison du fibrinogène. De plus, tout comme les autres inhibiteurs directs de la thrombine que sont la lépirudine et l'argatroban, elle se lie à la fois à la thrombine libre et à la thrombine liée au caillot de fibrine, contrairement à l'héparine (Figure). Elle forme un complexe, qui neutralise la thrombine durant la coagulation et la formation du thrombus. La bivalirudine 


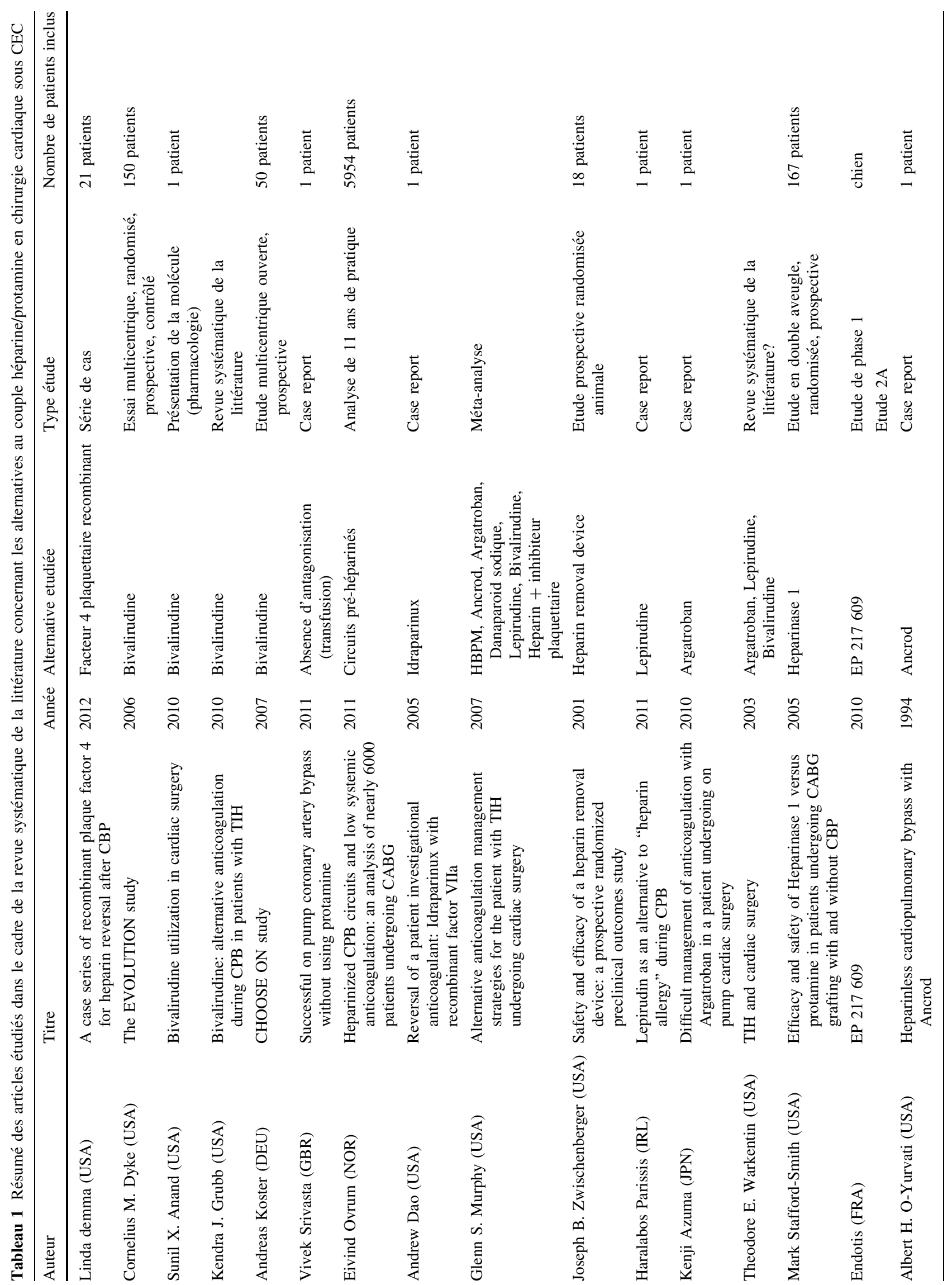




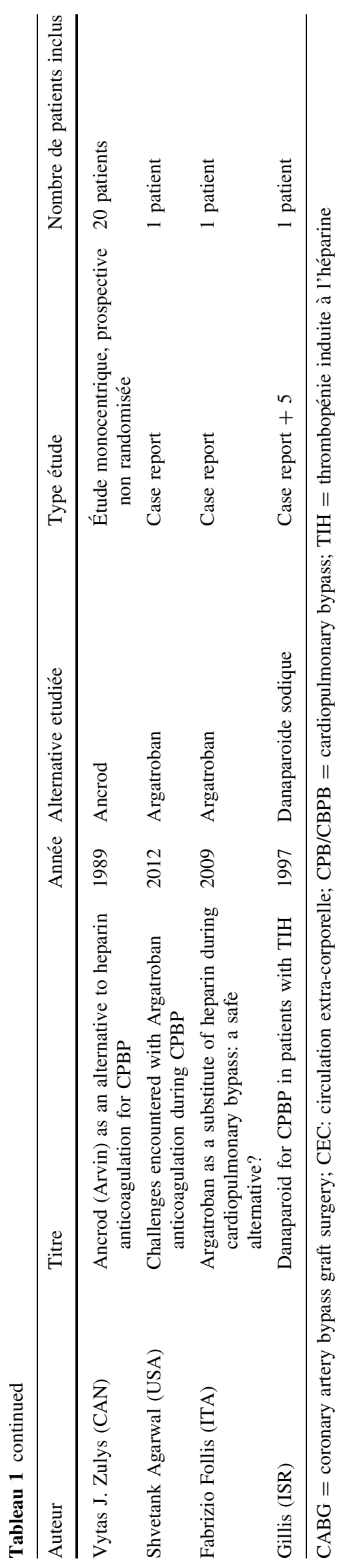

inhibe ainsi le clivage protéolytique du fibrinogène, l'activation des facteurs $\mathrm{V}$ et VIII, ainsi que l'activation plaquettaire induite par la thrombine. ${ }^{21}$

Son action anticoagulante se traduit par un allongement du temps de thromboplastine (TP) et une augmentation de l'INR (international normalized ratio) du ACT, du temps de thromboplastine partielle activée (aPTT) et du temps de thrombine (TT). ${ }^{21}$

Bien que la bivalirudine ait une AMM aux Etats-Unis pour une utilisation chez les patients à haut risque de thrombopénie à l'héparine devant avoir une angioplastie coronaire percutanée, ${ }^{23}$ l'utilisation en chirurgie cardiaque reste sujet à débat. Un pas a été franchi par la direction générale de la protection de la santé canadienne, qui recommande son utilisation pour ce même groupe de patients à risque nécessitant une angioplastie percutanée ou une chirurgie cardiaque. ${ }^{20}$

De nombreuses études, répertoriées dans le Tableau 3, se sont penchées sur l'efficacité et la sécurité de la bivalirudine par rapport à l'héparine non fractionnée chez des petites cohortes de patients à risque de TIH devant avoir une chirurgie cardiaque. ${ }^{20}$

En 2006, l'étude EVOLUTION-ON, qui est une étude randomisée, prospective, multicentrique, contrôlée, a comparé l'efficacité et la sécurité d'utilisation de la bivalirudine à la méthode d'anticoagulation de référence (héparine non fractionnée/protamine) chez 150 patients opérés de chirurgie cardiaque sous CEC (pontage aorto-coronarien, remplacement valvulaire). ${ }^{8}$ Le critère de jugement principal était le taux de succès de l'intervention défini par la survie des patients, l'absence d'infarctus du myocarde, d'accident vasculaire cérébral ou de la nécessité d'une nouvelle revascularisation. Les 101 patients randomisés dans le groupe bivalirudine reçurent un bolus intraveineux correspondant à $1 \mathrm{mg} \cdot \mathrm{kg}^{-1}$ suivi d'une perfusion continue de $2,5 \mathrm{mg} \cdot \mathrm{kg}^{-1} \cdot \mathrm{h}^{-1}$, afin d'obtenir un objectif d'ACT supérieur à 2,5 fois le témoin. Les résultats montrèrent un taux de succès comparable entre les deux stratégies ${ }^{8}$ (Tableau 3). L'utilisation de la bivalirudine est associée à un délai plus long entre la fin de la CEC et la fermeture de la paroi thoracique (71.2 vs. $56.6 \mathrm{~min}, P=0.015)$, sans différence statistiquement significative en terme de temps opératoire total. De plus, cette alternatives est associée à un saignement postopératoire précoce (deux heures) statistiquement supérieur $(238 \mathrm{~mL} \quad$ vs. $160 \mathrm{~mL}$; $P=0.0009)$ sans différence statistiquement significative à $24 \mathrm{~h}(793 \mathrm{~mL}$ vs. $668 \mathrm{~mL} ; P=0.15){ }^{8}$

Cette étude a montré que la bivalirudine pouvait être un anticoagulant sûr et efficace pour les patients devant subir une chirurgie cardiaque sous CEC.

L'étude CHOOSE-ON, quant à elle, est une étude multicentrique, prospective, ouverte publiée en 2007 


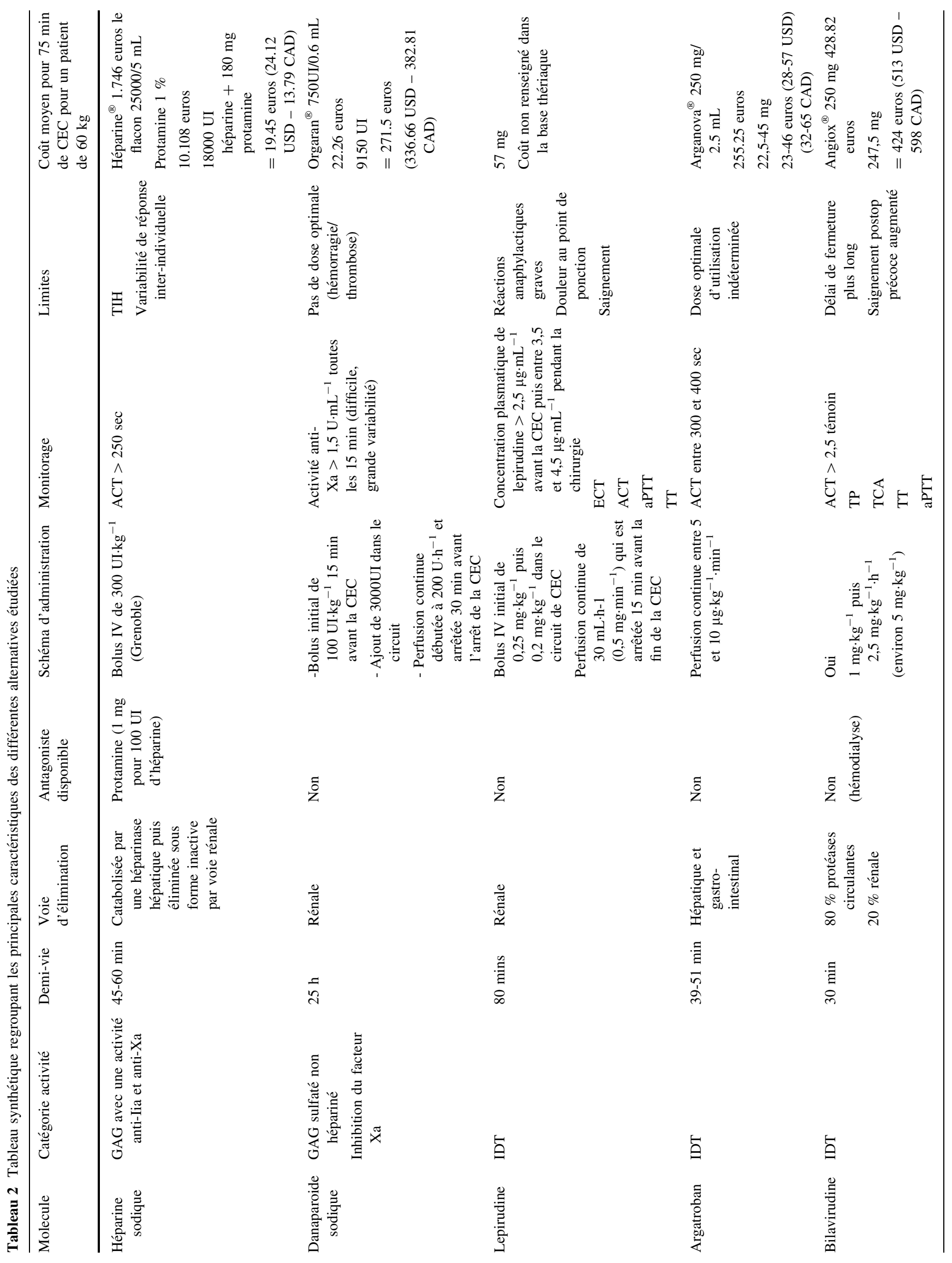




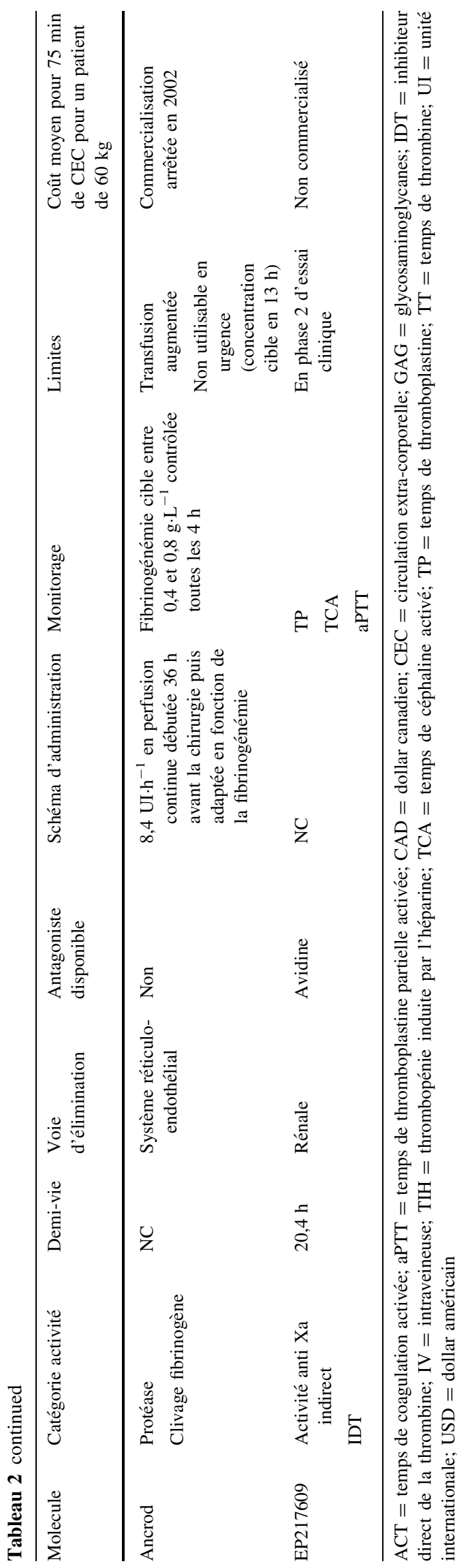

incluant 50 patients de chirurgie cardiaque sous CEC ayant un antécédent de $\mathrm{TIH}^{22}$ Les résultats allaient dans le même sens que l'étude EVOLUTION-ON.

Certaines études tendent à montrer que l'utilisation de la bivalirudine améliorerait angiographiquement la perméabilité et le flux dans les vaisseaux pontés lors d'un pontage aorto-coronarien. $^{22}$

Sur le plan économique, un flacon de $250 \mathrm{mg}$ de bivalirudine coûte 428.82 euros (531 USD- 600 CAD). Si on se réfère au protocole utilisé dans l'étude EVOLUTION-ON, le coût moyen de cette alternative serait de 424 euros (526 USD -593 CAD) pour un patient de $60 \mathrm{~kg}$, soit plus de 20 fois plus que la stratégie de référence.

Une seconde limite à l'utilisation de la bivalirudine est le risque de précipitation avec un certain nombre de médicaments: altéplase, amiodarone, amphotéricine B, chlorpromazine, diazépam, édisylate de prochlorpérazine, rétéplase, streptokinase, vancomycine. ${ }^{24}$

\section{Ancrod}

L'ancrod est une protéase, dérivée du vénin d'une vipère de Malaisie (Calloselasma rhodostoma). Elle permet une diminution de la concentration de fibrinogène via le clivage $\mathrm{du}$ fibrinogène $\mathrm{A}$ et est ensuite éliminée par le système réticuloendothélial en plusieurs jours.

L'Ancrod a été utilisé avec succès dans plusieurs cas de chirurgie cardiaque sous CEC, notamment chez des patients présentant des thrombopénies induites à l'héparine dans les années $1980 .^{25,26}$ Une perfusion continue préopératoire de $1,65 \mathrm{UI} \cdot \mathrm{kg}^{-1}$ en $13 \mathrm{~h}$ a permis d'obtenir une concentration cible de fibrinogène entre 0,4 et $0,8 \mathrm{~g} \cdot \mathrm{L}^{-1}$. Ces études de cas rapportent néanmoins une consommation plus importante de produits sanguins. Ancrod est malgré cela difficilement utilisable en chirurgie cardiaque en raison de son délai d'action; de plus, elle n'inhibe pas la formation de thrombine, son administration doit être lente en raison de dépôt de fibrine intra-vasculaire et aucun moyen de réversion fiable n'est disponible à ce jour. ${ }^{1}$ Cette molécule n'est plus commercialisée en France depuis 2002.

\section{Idraparinux}

De grandes avancées ont été faites depuis la découverte du mécanisme d'action de l'héparine au niveau de la thrombine. En effet, une petite séquence de pentasaccharides compose le site de liaison à la thrombine avec une activité anti-Xa spécifique. ${ }^{27}$ En se basant sur cette séquence de pentasaccharides, la synthèse de molécules avec des profils pharmacodynamiques hautement spécifiques a pu être réalisée telle que le fondaparinux. La molécule du fondaparinux est quasiment 


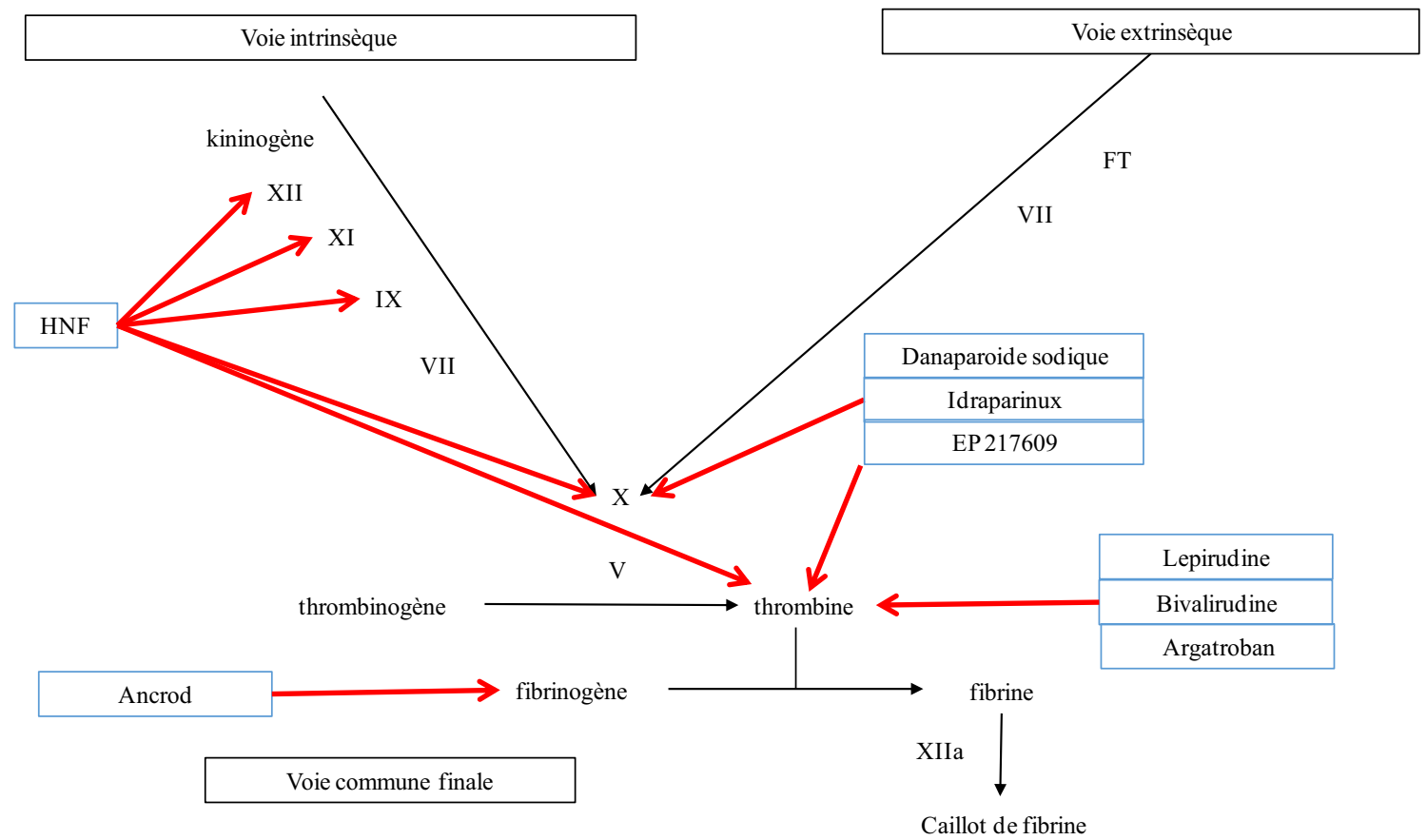

Figure Représentation schématique du mode d'anticoagulation de l'héparine non fractionnée (HNF) et de la bivalirudine. Reproduit avec la permission de: Anand SX, Viles-Gonzalez JF, Mahboobi SK, Heerdt $P M$. Bivalirudin utilization in cardiac surgery: shifting

anticoagulation from indirect to direct thrombin inhibition. Can $\mathrm{J}$ Anesth 2011; 58: 296-311. ${ }^{20} \mathrm{HNF}=$ héparine non fractionnée; $\mathrm{FT}=$ facteur tissulaire

Tableau 3 Principales études en chirurgie cardiaque comparant l'anticoagulation entre la bivalirubine et l'HNF

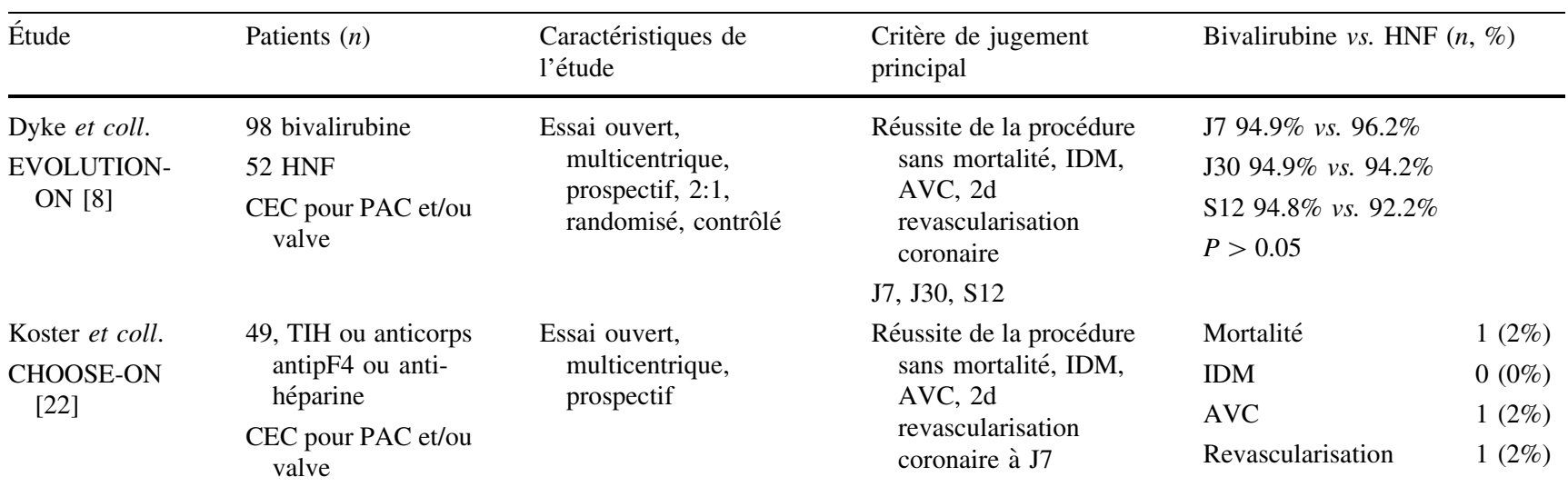

antipF4 = anti facteur 4 plaquettaire, $\mathrm{AVC}=$ accident vasculaire cérébral, $\mathrm{CEC}=$ circulation extra-corporelle, $\mathrm{HNF}=$ héparine non fractionnée, IDM = infarctus du myocarde, PAC = pontage aorto-coronarien, TIH = Thrombopénie induite par l'héparine. Reproduit avec la permission de: Anand SX, Viles-Gonzalez, JF, Mahboobi SK, Heerdt PM. Bivalirudin utilization in cardiac surgery: shifting anticoagulation from indirect to direct thrombin inhibition. Can J Anesth 2011; 58: 296-311 20

identique à la séquence de pentasaccharide correspondant au site de liaison de l'héparine sur la thrombine avec une activité anti-Xa environ 300 fois supérieur. ${ }^{27}$ L'Idraparinux est un analogue du fondaparinux avec une plus grande affinité pour la thrombine. L'Idraparinux a une demi-vie d'élimination de $120 \mathrm{~h}$ après une injection sous-cutanée, ce qui en fait un anticoagulant difficilement utilisable en chirurgie cardiaque, d'autant plus qu'il ne se lie pas à la thrombine liée au caillot. ${ }^{18}$

\section{EP217609}

Dans la lignée de l'idraparinux, des molécules telles que l'EP42675 puis l'EP 271609 ont été synthétisées. L'EP 
217609 est un nouvel anticoagulant parentéral de synthèse, qui présente les caractéristiques suivantes: ${ }^{27}$

- Il est à la fois un inhibiteur indirect du facteur Xa et un inhibiteur direct de la thrombine (Figure).

- Il inhibe à la fois la thrombine libre et la thrombine liée au caillot et ceci indépendamment de l'antithrombine.

- Il a une élimination principalement rénale.

- Il a un effet dose dépendant avec les principaux tests de coagulation (aPTT, TP, TCT).

- Il n'a pas de réactivité croisée avec les anticorps anti PF4.

- Il est quasiment instantanément neutralisable par l'avidine, une glycoprotéine dérivée de l'œuf.

Cette nouvelle molécule a fait l'objet d'une étude de phase I chez 40 sujets sains. ${ }^{28}$ Cette étude montre un pic de concentration après l'injection intraveineuse avec une faible variabilité entre les sujets et une bonne corrélation entre les doses injectées et les principaux tests de la coagulation que sont l'ACT, le TT ou l'activité anti-Xa. Aucun effet indésirable ne semble avoir été observé après un suivi de dix jours.

Une étude NOUVELLE de phase IIa a été débutée en 2011 par le laboratoire ENDOTIS chez 36 patients subissant une chirurgie cardiaque. Cette étude a été interrompue en 2012 et les résultats ne sont pas connus.

\section{Discussion}

L'utilisation de l'héparine et de la protamine reste la stratégie d'anticoagulation de référence en chirurgie cardiaque sous CEC. Cependant, certaines situations nécessitent d'utiliser d'autres molécules en tant qu'alternative d'anticoagulation et de mettre en place des procédures avec des schémas d'utilisation fiables et validés.

Plusieurs molécules ont fait l'objet d'étude avec des niveaux de preuve scientifique variables (case report, étude randomisée, méta-analyse,...). L'anticoagulant idéal pouvant être utilisé en chirurgie cardiaque sous CEC n'existe pas à l'heure actuelle.

La bivalirudine semble être la molécule la plus prometteuse à ce jour, du moins celle dont les études semblent les plus robustes. Elle présente l'avantage d'avoir à la fois un délai d'action rapide, une courte demi-vie d'action et un mécanisme d'élimination basé principalement sur des protéases circulantes. Le protocole d'utilisation présenté dans le Tableau 2 parait engendrer peu de variations interindividuelles contrairement aux autres molécules étudiées. Ses principales limites sont l'absence d'antagoniste disponible à l'heure actuelle ainsi que son coût d'utilisation élevé (plus de 20 fois supérieur à l'héparine).
Les autres alternatives présentées dans cette revue systématique de la littérature ont comme principal inconvénient le fait de n'avoir été étudiées que chez un nombre restreint de patients, dans le cadre d'étude de faible niveau de preuve (case report,...) avec l'absence de protocole reproductible. De plus, la majorité de ces anticoagulants représente un coût nettement supérieur au coût de l'anticoagulation de référence. Ceci représente une limite d'utilisation majeure de ses molécules compte tenu de la politique de santé actuelle. Certains anticoagulants telles que l'ancrod semblent désuètes et ne sont d'ailleurs plus commercialisée à l'heure actuelle en France.

Cette revue systématique de la littérature présente probablement certaines limites méthodologiques: la première est l'absence d'exhaustivité possible de la recherche car de il est probable que des études appropriées sur ce sujet n'aient pas été publiées. De plus, certaines publications sélectionnées pour cette analyse ne possèdent sont de faible niveau de preuve (case report) et ne répondent donc pas au standard de qualité tel que défini dans la seconde étape de la Cochrane Collaboration Handbook. ${ }^{5}$ Ce travail a tenté de donner une approche globale de l'ensemble des alternatives d'anticoagulation connue à l'heure actuelle mais la poursuite de recherche de nouveaux anticoagulants pouvant être utilisé en chirurgie cardiaque sous CEC est primordial. De nouvelles molécules telles que l'EP 217609 semblent séduisantes mais restent encore au stade d'essai clinique de phase II.

En conclusion, la recherche d'alternatives fiables au couple héparine/protamine en chirurgie cardiaque devient un enjeu de santé publique compte tenu de l'existence de contre-indications indiscutables à l'héparine (TIH) mais aussi de scandales sanitaires (héparine contaminée, risque de pénurie suite à l'accident de Fukushima). Plusieurs alternatives ont fait l'objet d'essai clinique avec des résultats plus ou moins satisfaisants. De nouvelles études solides sont indispensables afin de définir un protocole fiable et reproductible en alternative à la stratégie de coagulation actuelle.

Conflit d'intérêt Aucun.

\section{Références}

1. Murphy GS, Marymont JH. Alternative anticoagulation management strategies for the patient with heparin-induced thrombocytopenia undergoing cardiac surgery. J Cardiothorac Vasc Anesth 2007; 21: 113-26.

2. Warkentin TE, Greinacher A. Heparin-induced thrombocytopenia and cardiac surgery. Ann Thorac Surg 2003; 76: 2121-31.

3. Park $K W$. Protamine and protamine reactions. Int $\mathrm{J}$ Anesthesiol Clin 2004; 42: 135-45. 
4. Kimmel SE, Sekeres M, Berlin JA, Ellison N. Mortality and adverse events after protamine administration in patients undergoing cardiopulmonary bypass. Anesth Analg 2002; 94: 1402-8.

5. Montpas $N$, Desormeaux A, Keire D, Adam A. Les réactions anaphylactoïdes associées à l'héparine d'origine chinoise. Ann Pharm Fr 2011; 69: 258-64.

6. Le Meur N. Alerte Produit. Lettre d'information à l'attention des pharmaciens hospitaliers. 2013; Disponible à l'adresse URL: http://sofia.medicalistes.org/spip/IMG/pdf/Lettre_du_laboratoire_ sanofi-aventis_France_aux_professionnels_de_sante_concernes. pdf (consulté janvier 2015).

7. Higgins J, Churchill R, Cumpston M, Chandler J S. Cochrane Handbook for Systematic Reviews of Interventions Version 5.1.0. 2011. Disponible à l'adresse URL: http://www.cochrane.org/ handbook (consulté janvier 2015).

8. Dyke CM, Smedira NG, Koster A, et al. A comparison of bivalirudin to heparin with protamine reversal in patients undergoing cardiac surgery with cardiopulmonary bypass: the EVOLUTION-ON study. J Thorac Cardiovasc Surg 2006; 131: 533-9.

9. Doherty DC, Ortel TL, de Bruijn N, Greenberg CS, Van Trigt $P$ 3rd. "Heparin-free" cardiopulmonary bypass: first reported use of heparinoid (Org 10172) to provide anticoagulation for cardiopulmonary bypass. Anesthesiology 1990; 73: 562-5.

10. Grocott HP, Root J, Berkowitz SD, deBruijn N, Landolfo $K$. Coagulation complicating cardiopulmonary bypass in a patient with heparin-induced thrombocytopenia receiving the heparinoid, danaparoid sodium. J Cardiothorac Vasc Anesth 1997; 11: 875-7.

11. Gillis $S$, Merin $G$, Zahger $D$, et al. Danaparoid for cardiopulmonary bypass in patients with previous heparininduced thrombocytopenia. Br J Haematol 1997; 98: 657-9.

12. Magnani HN, Beijering RJ, ten Cate JW, Chong BH. Orgaran anticoagulation for cardiopulmonary bypass in patients with heparin-induced thrombocytopenia. In: Pifarre RE, editor. New Anticoagulants for the Cardiovascular Patient. Philadelphia, PA: Hanley \& Belfus; 1997. p. 487-500.

13. Grubb KJ, Salehi P, Chedrawy EG. Bivalirudin: alternative anticoagulation during cardiopulmonary bypass in patients with heparin-induced thrombocytopenia. Recent Pat Cardiovasc Drug Discov 2010; 5: 20-4.

14. Glusa E, Markwardt F. Platelet functions in recombinant hirudinanticoagulated blood. Haemostasis 1990; 20: 112-8.

15. Parissis $H$. Lepirudin as an alternative to "heparin allergy" during cardiopulmonary bypass. J Cardiothorac Surg 2011; 6: 44.

16. Greinacher A, Lubenow $N$, Eichler $P$. Anaphylactic and anaphylactoid reactions associated with lepirudin in patients with heparin-induced thrombocytopenia. Circulation 2003; 108 : 2062-5.
17. Azuma K, Maruyama K, Imanishi H, Nakagawa H, Kitamura A, Hayashida $M$. Difficult management of anticoagulation with argatroban in a patient undergoing on-pump cardiac surgery. J Cardiothorac Vasc Anesth 2010; 24: 831-3.

18. Follis $F$, Filippone $G$, Montalbano $G$, et al. Argatroban as a substitute of heparin during cardiopulmonary bypass: a safe alternative? Interact Cardiovasc Thorac Surg 2010; 10: 592-6.

19. Agarwal S, Ullom B, Al-Baghdadi Y, Okumura M. Challenges encountered with argatroban anticoagulation during cardiopulmonary bypass. J Anaesthesiol Clin Pharmacol 2012; 28: 106-10.

20. Anand SX, Viles-Gonzalez JF, Mahboobi SK, Heerdt PM. Bivalirudin utilization in cardiac surgery: shifting anticoagulation from indirect to direct thrombin inhibition. Can J Anesth 2011; 58: 296-311.

21. Warkentin TE, Greinacher A, Koster A. Bivalirudin. Thromb Haemost 2008; 99: 830-9.

22. Koster A, Dyke CM, Aldea $G$, et al. Bivalirudin during cardiopulmonary bypass in patients with previous or acute heparin-induced thrombocytopenia and heparin antibodies: results of the CHOOSE-ON trial. Ann Thorac Surg 2007; 83: $572-7$.

23. Lincoff AM, Bittl JA, Harrington RA, et al. Bivalirudin and provisional glycoprotein IIb/IIIa blockade compared with heparin and planned glycoprotein IIb/IIIa blockade during percutaneous coronary intervention: REPLACE-2 randomized trial. JAMA 2003; 289: 853-63.

24. Centre hospitalier universitaire Vaudois (CHUV). Commission permanente des médicaments. Bulletin d'information CPM No 4, 2007. Utilisation de la bivalirudine (Angiox) au CHUV. Disponible à l'adresse URL: http://www.chuv.ch/pha/pha_home/ pha-medicament/pha-medicament-prescription/pha-medicamentprescription-recommandations.htm (consulté janvier 2015).

25. Ah OY, Laub GW, Southgate TJ, McGrath LB. Heparinless cardiopulmonary bypass with ancrod. Ann Thorac Surg 1994; 57: 1656-8.

26. Zulys VJ, Teasdale SJ, Michel ER, et al. Ancrod (Arvin) as an alternative to heparin anticoagulation for cardiopulmonary bypass. Anesthesiology 1989; 71: 870-7.

27. Petitou M, Nancy-Portebois V, Dubreucq G, et al. From heparin to EP217609: the long way to a new pentasaccharide-based neutralisable anticoagulant with an unprecedented pharmacological profile. Thromb Haemost 2009; 102: 804-10.

28. Gueret P, Krezel C, van Giersbergen PL, Fuseau E, Petitou M, Neuhart E. First human study with EP217609, a new synthetic parenteral neutralizable dual action anticoagulant. ICT 2010: 177 (résumé). 